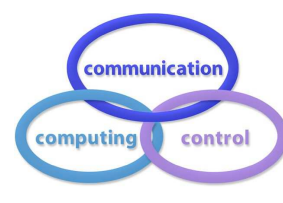

\title{
CCC Publications
}

\section{Trust Based Fuzzy Linguistic Recommender Systems as Reinforcement for Personalized Education in the Field of Oral Surgery and Implantology}

\author{
C. Porcel, J. Herce-Zelaya, J. Bernabé-Moreno, A. Tejeda-Lorente, E. Herrera-Viedma
}

\author{
Carlos Porcel* \\ Department of Computer Science \\ University of Jaén, Spain \\ Campus Las Lagunillas s/n. 23071, Jaén, Spain \\ *Corresponding author: cporcel@ujaen.es
}

\section{Julio Herce-Zelaya}

Andalusian Research Institute in Data Science and Computational Intelligence, University of Granada, Granada 18071, Spain

julioherce@gmail.com

\section{Juan Bernabé-Moreno}

Andalusian Research Institute in Data Science and Computational Intelligence, University of Granada, Granada 18071, Spain jbernabemoreno@gmail.com

\section{Alvaro Tejeda-Lorente}

Andalusian Research Institute in Data Science and Computational Intelligence, University of Granada, Granada 18071, Spain atejeda@decsai.ugr.es

\section{Enrique Herrera-Viedma}

Andalusian Research Institute in Data Science and Computational Intelligence, University of Granada, Granada 18071, Spain viedma@decsai.ugr.es

\begin{abstract}
The rapid advances in Web technologies are promoting the development of new pedagogic models based on virtual teaching. In this framework, personalized services are necessary. Recommender systems can be used in an academic environment to assist users in their teaching-learning processes. In this paper, we present a trust based recommender system, adopting a fuzzy linguistic modeling, that provides personalized activities to students in order to reinforce their education, and applied it in the field of oral surgery and implantology. We don't take into account users with similar ratings history but users in which each user can trust and we provide a method to aggregate the trust information. This system can be used in order to aid professors to provide students with a personalized monitoring of their studies with less effort. The results obtained in the experiments proved to be satisfactory.
\end{abstract}

Keywords: recommender system, e-learning, fuzzy linguistic modeling, oral surgery. 


\section{Introduction}

The great advances in Web technologies are promoting the development of new pedagogic models that complement the present education [11]. The new technologies improve the teaching-learning processes, aiding the information broadcasting in an efficient and easy manner, and providing tools for the personal and global communications that allow encouraging the collaborative learning $[3,19]$. In this academic scope, personalized education [16] can be very helpful aiding students to reinforce the areas where it is necessary some help as well as maximizing those where they have potential. Education must also have the ability to adapt itself to the necessities of the student dynamically.

Recommender systems seek to discover information items that are valuable to the users. They may be considered personalized services because they have an independent profile for each user $[2,4$, 20]. Therefore these systems need some information about every user, such as the ratings provided by the users about the analyzed items. This need for information introduces the requirement for the system to maintain users' profiles containing the users' preferences or needs. Another aspect to take into consideration is which additional information is required by the system, and how this information is processed and managed to generate a list of personalized recommendations. One of the mostly used methods to generate recommendations is the collaborative approach in which the recommendations provided to a particular user are based upon the ratings provided by those users with similar profiles. In this sense, we can use the inherent connectivity from an educational community to support collaborative approach, where students rate resources and these ratings are shared with a large community $[3,4]$.

One key disadvantage of this approach consists of the need of many ratings to obtain a good performance. But users typically provide just a few ratings, so the systems have difficulties to compute the similarity between two users [14]. Therefore, collaborative approaches tend to fail in generating recommendations since they usually fail at obtaining groups of users with similar preferences. Thus, some improvements need to be introduced to overcome this situation and one promising direction is to focus on trust, which plays a crucial role in on-line social networks [5], so widespread and popular today. People tend to rely upon recommendations received from trusted users, such as friends, more than those generated by automatic systems [18]. In the literature, we can find some proposals about the incorporation of trust models in recommendation systems $[10,21]$. In these systems, the recommendation engine uses the trusted network between users.

The aim of this article is to present a new fuzzy linguistic recommender system that incorporates the concept of trust in the recommendations generation engine. This new system is also adapted to students of Dentistry from the Dentistry School of the University of Granada ${ }^{1}$ (Spain). The major innovations and contributions of the system include:

1. A method to estimate the trust score between two users, because the trusted network can be huge and most users do not know each other.

2. The provision of reliable personalized information by using a recommendation approach in which users with similar ratings history or pedagogical needs are not considered, but users in which each user can trust.

3. Its user-friendly nature, using a multi-granular fuzzy linguistic modeling to improve the representation of user preferences and facilitate user-system interactions $[9,12]$.

4. The ability to use it in any place and at any time, providing to students the necessary freedom to organize their schedules.

5. The reliability of the information offered and the selection of exercises, endorsed by a team of experts in oral surgery from the Dentistry School of the University of Granada.

The paper is structured as follows. In Section 2, the preliminaries are presented. Next in Section 3 , we describe our proposal. Section 4 addresses the evaluation of the system and finally in Section 5 we throw our conclusions.

\footnotetext{
${ }^{1}$ www.ugr.es
} 


\section{Preliminaries}

\subsection{Basis of recommender systems}

Recommender systems try to guide the user in a personalized way towards suitable tasks among a wide range of possible options $[2,20]$. In order to generate personalized recommendations that are tailored to the user's preferences or needs, recommender systems must collect personal preference information. Taking into account the knowledge source, different recommendations generation methods can be distinguished $[1,14]$. Each approach has certain advantages and disadvantages, depending on the scope settings. One solution is to use a hybrid strategy combining different approaches in order to reduce the disadvantages of each one of them and to exploit their benefits[1]. Moreover, the recommendation activity is followed by a feedback phase in which the users provide the system with their satisfaction evaluations about the recommended items and the system uses these evaluations to automatically update user profiles.

\subsection{Fuzzy linguistic approach}

The fuzzy linguistic approach is a tool based on the concept of linguistic variable proposed by Zadeh [22]. This theory has given very good results to model qualitative information and it has been proven to be useful in many problems.

In [6] is proposed a continuous model of information representation based on 2-tuple fuzzy linguistic modelling. To define it both the 2 -tuple representation model and the 2-tuple computational model to represent and aggregate the linguistic information have to be established. Let $S=\left\{s_{0}, \ldots, s_{g}\right\}$ be a linguistic term set with odd cardinality. We assume that the semantics of labels is given by means of triangular membership functions and consider all terms distributed on a scale on which a total order is defined. If a value $\beta \in[0, g]$, and $\beta \notin\{0, \ldots, g\}$, we can represent $\beta$ as a 2 -tuple $\left(s_{i}, \alpha_{i}\right)$, where $s_{i}$ represents the linguistic label, and $\alpha_{i}$ is a numerical value expressing the value of the translation between numerical values and 2-tuple: $\Delta(\beta)=\left(s_{i}, \alpha\right)$ and $\Delta^{-1}\left(s_{i}, \alpha\right)=\beta \in[0, g][6]$. In order to establish the computational model negation, comparison and aggregation operators are defined. Using functions $\Delta$ and $\Delta^{-1}$, any of the existing aggregation operators (such as arithmetic mean, weighted average operator or linguistic weighted average operator) can be easily be extended for dealing with linguistic 2-tuples without loss of information [6].

When different experts have different uncertainty degrees on the phenomenon or when an expert has to evaluate different concepts, several linguistic term sets with a different granularity of uncertainty are necessary. To manage this situation, in [7] a multi-granular 2-tuple fuzzy linguistic modelling based on the concept of linguistic hierarchy is proposed. A Linguistic Hierarchy LH, is a set of levels $l(t, n(t))$, where each level $t$ is a linguistic term set with different granularity $n(t)$. In [7] a family of transformation functions between labels from different levels was introduced. To establish the computational model we select a level that we use to make the information uniform and thereby we can use the defined operator in the 2 -tuple model. This result guarantees that the transformations between levels of a linguistic hierarchy are carried out without loss of information.

\subsection{Trust networks}

Trust networks are social networks in which users can explicitly assign trust scores to rate other users. But trust networks are usually very large and therefore a lot of users don't even know the vast majority of other users. For this reason, we need to use a method to estimate the trust degree between two users. The idea is to search for a path between the two users and propagate the trust degrees found along the path. Usually, we can find several paths between two users, so we may select the most relevant and aggregate the propagated trust degrees into the trust degree estimation. An upper path length limit is typically imposed what is known as horizon, $H$, and typical values for $H$ are 2 or 3 .

To aggregate the propagated trust degrees of the paths found, we use MILOWA, a majority guided linguistic operator [8]. With respect to the variable inducing the reordering of the set of values to be aggregated, in a trust network we work with information about reliability, so that we use the average 
global trust of all users of each of the founded path. To compute the global trust of a user we use PageRank [13] because is one of the most widely used global trust metric.

\section{Trust based recommender system to assist dentistry students in the field of oral surgery and implantology}

In this section we present the Web system to assist students from the Dentistry School of University of Granada. Initially it is oriented to students from these subjects: Oral Surgery I, Oral Surgery II and Implantology. The system has three main components: videos and resources, student profiles and the method for generating recommendations.

\subsection{Information representation}

In order to allow for higher flexibility in the communication processes of the system, different label sets $\left(S_{1}, S_{2}, \ldots\right)$ are used are selected from among those that compose a $L H$, i.e., $S_{i} \in L H$. The different concepts assessed in the system are the following:

- Degree of trust of a student relative to another, which is labelled in $S_{1}$.

- The predicted degree of relevance of a resource for a student, which is labeled in $S_{2}$.

- The degree of satisfaction with a recommended resource expressed by a student, which is labeled in $S_{3}$.

- Membership degree of a resource scope or student needs with respect to each of the defined reinforcing subgroup, which is labelled in $S_{4}$.

We use 5 labels to represent the degrees of trust, satisfaction and membership to reinforcing subgroup $\left(S_{1}=S^{5}, S_{3}=S^{5}\right.$ and $\left.S_{4}=S^{5}\right)$ and 9 labels to represent the predicted relevance degrees $\left(S_{2}=S^{9}\right)$. The linguistic terms in each level are the following ones:

- $S^{5}=\left\{b_{0}=N o n e=N, b_{1}=\right.$ Low $=L, b_{2}=$ Medium $=M, b_{3}=$ High $=H, b_{4}=$ Total $\left.=T\right\}$

- $S^{9}=\left\{c_{0}=\right.$ None $=N, c_{1}=$ Very_Low $=V L, c_{2}=$ Low $=L, c_{3}=$ More_Less_Low $=$ $M L L, c_{4}=$ Medium $=M, c_{5}=$ More_Less_High $=M L H, c_{6}=$ High $=\bar{H}, c_{7}=$ Very_High $=V H, c_{8}=$ Total $\left.=T\right\}$

\subsection{Resource representation}

A multimedia database was developed and contained videos with a wide set of different oral surgeries or implants for all possible needs inside the subjects covered. Also, different sets of scientific papers or class notes are introduced into the system. All videos, papers and notes can be combined among different subgroups, called activities, in the construction of a customized program for each student. Videos were recorded on real surgeries produced in dentistry's offices or in university's labs. Those activities are the items to be recommended by our system. Each combination of videos, notes or papers make the different activities suitable for a student with a specific pedagogical need.

Once a teacher creates a new activity into the system, he/she provides the activity with an internal representation that is mainly based on its appropriateness for each reinforce subgroup. An activity $i$ is represented as a vector $V R_{i}=\left(V R_{i 1}, V R_{i 2}, \ldots, V R_{i 4}\right)$, where each component $V R_{i j} \in S_{4}$ is a linguistic assessment that represents how appropriate is the activity $i$ with respect to the reinforcing subgroup $j$. 


\subsection{Student profiles}

The student profiles are represented by three components: their needs, their degrees of trust in other students and the satisfaction degrees with the recommended resources. Students must complete their profiles with the grades obtained in previous subjects related with this oral surgery and implantology. They have to periodically carry out different test to be able of evaluate their abilities. After obtaining the test results, the teachers assess the membership of the student need in each one of the four reinforcing subgroups. A student $i$ is represented as a vector $V S_{i}=\left(V S_{i 1}, V S_{i 2}, \ldots, V S_{i 4}\right)$, where each component $V S_{i j} \in S_{4}$ is a linguistic assessment that represents the degree of how appropriate $i$ is for each reinforcing subgroup $j$. Since student are performing test over the whole semester, their membership to the different subgroup will be changing together with their new results. Besides, the students explicitly specify their degree of trust on other students using the level $S_{1}$ of the $L H$, i.e., using one the 5 labels of $S^{5}$. Finally, as students receive recommendations they are asked to assess their satisfaction with the recommendations $\left(r c \in S_{3}\right)$.

\subsection{Recommendation approach}

As a large number of users have not supplied the trust degrees to many other users, to generate recommendations, we need a method to estimate the trust degree between users. Then, in order to estimate the level in which a user $u$ trust in other user $v, \tau_{u, v}$, the MLIOWA operator (see Section 2.3 ) is applied to aggregate the global trust of all users found in the several paths between $u$ and $v$, according to the majority [8].

Then, if we wish to estimate or upgrade the relevance of a item $i$ for a user $u$, the following steps are performed:

1. Identify the set of trusted users of $u, \Gamma_{u}$. To do that, we estimate the trust between $u$ and all other users taking into account the selected horizon, i.e. $\tau_{u, v} \forall v \in \Upsilon$ with $v \neq u$ and $\Upsilon$ the set of users. As $S_{1}=S^{5}$, we consider that the user $v$ is a trusted user of $u$ if the trust degree is higher than the mid linguistic label.

2. To recovery the assessments provided by the trusted users of $u$ over the item $i$, i.e., the linguistic satisfaction assessments $\operatorname{sat}(y, i) \in S_{3}, \forall y \in \Gamma_{u}$.

3. The item $i$ is recommended to $u$ with a predicted relevance degree $p_{\text {rel }}(u, i) \in S_{2} \times[-0.5,0.5]$ which is calculated as follows:

$$
p_{r e l}(u, i)=\bar{x}_{l}^{w}\left(\left(T F_{S_{2}}^{S_{3}}\left(\operatorname{sat}\left(y_{1}, i\right), 0\right), T F_{S_{2}}^{S_{1}}\left(\tau_{u, y_{1}}\right)\right), \ldots,\left(T F_{S_{2}}^{S_{3}}\left(\operatorname{sat}\left(y_{n}, i\right), 0\right), T F_{S_{2}}^{S_{1}}\left(\tau_{u, y_{n}}\right)\right)\right),
$$

where $y_{1}, \ldots, y_{n} \in \Gamma_{u}, \bar{x}_{l}^{w}$ is the linguistic weighted average operator and $T F_{t^{\prime}}^{t}$ is the transformation function between a 2-tuple that belongs to level $t$ and another 2-tuple in level $t^{\prime} \neq t$.

\section{System evaluation}

\subsection{Validating the system utility}

In the first place, we performed a practical study where a group of 50 volunteers students tested the system during one semester in different subjects: Oral surgery I, Oral surgery II and Implantology $I$. Then, we evaluated the utility of the system based on the results obtained by the students and the feedback provided. After one semester of usage, the results of the students who used the system were in average a $15.5 \%$ better than the users that only assist to lessons. The linguistic weighted average of the feedback provided by users on the improvement received by the recommendations were $\left(b_{0}^{5}, 7\right)$, that is between medium and high. Therefore, the results demonstrate that the website is not only positively perceived by its users but also it increases their results compared to the ones who did not use it. 


\subsection{Recommendation approaches evaluation}

We also validated the proposed approach with off-line tests, where the Epinions dataset ${ }^{2}[10]$ was used. Due to performance reasons, we reduced the Epinion dataset containing a sample of the first 1000 users and 2000 items. The ratings subset for this reduced subset still contained 7841 ratings and 52548 trust statement values.

\subsubsection{Experiments description}

To develop the experiments we implemented the approach considering different values for the horizon, i.e., $H=2, H=3$ and $H=4$ (called Trust-H2, Trust-H3 and Trust-H4). In order to compare the results, we also implemented some collaborative approaches. We implemented both item-based and user-based approaches [1] with different configurations. We've also analysed the impact of having a different number of neighbours on the similarity computation, achieved by using different values for the variable $K$, which represents the most similar k-users. Specifically, we used following values for $K: 10,20$ and 50. In addition, we combined these values with two different similarity metrics, such as Cosine and Pearson. We name Col-It-or Col-Us- the algorithms item and user based respectively; likewise we added to the algorithm name the suffixes $C s$ or Ps for Cosine or Pearson metrics; the last suffix corresponds to the number of neighbours (K10, K20 or K50). Then, we carried out a 5-fold cross validation process [15] and to measure the accuracy, we adopted the Mean Absolute Error (MAE) [17].

On the other hand, we also analysed the coverage achieved with each approach [17], i.e., the proportion of ratings of the validation set the system can generate a prediction for, classifying users and items into different types [10]. The users are classified in these types: cold start users who provided from 1 to 4 ratings, heavy raters who provided more than 10 ratings, opinionated users who provided more than 4 ratings and whose standard deviation is greater than 1.5, black sheep users who provided more than 4 ratings and for which the average distance of their rating on corresponding item with respect to mean rating of the item is greater than 1 . The items are classified in: niche items which received less than 5 ratings and controversial items which received ratings whose standard deviation is greater than 1.5 .

\subsubsection{Experiments results}

Figure 1 shows respectively the MAE and coverage for cosine/Pearson similarity measure for itembased and users-based collaborative approaches for the different user groups. The MAE obtained is similar for all combinations (except for small differences), but the coverage is better with the userbased collaborative approach. In both cases, the higher the number of neighbours, the better the results, especially in coverage.
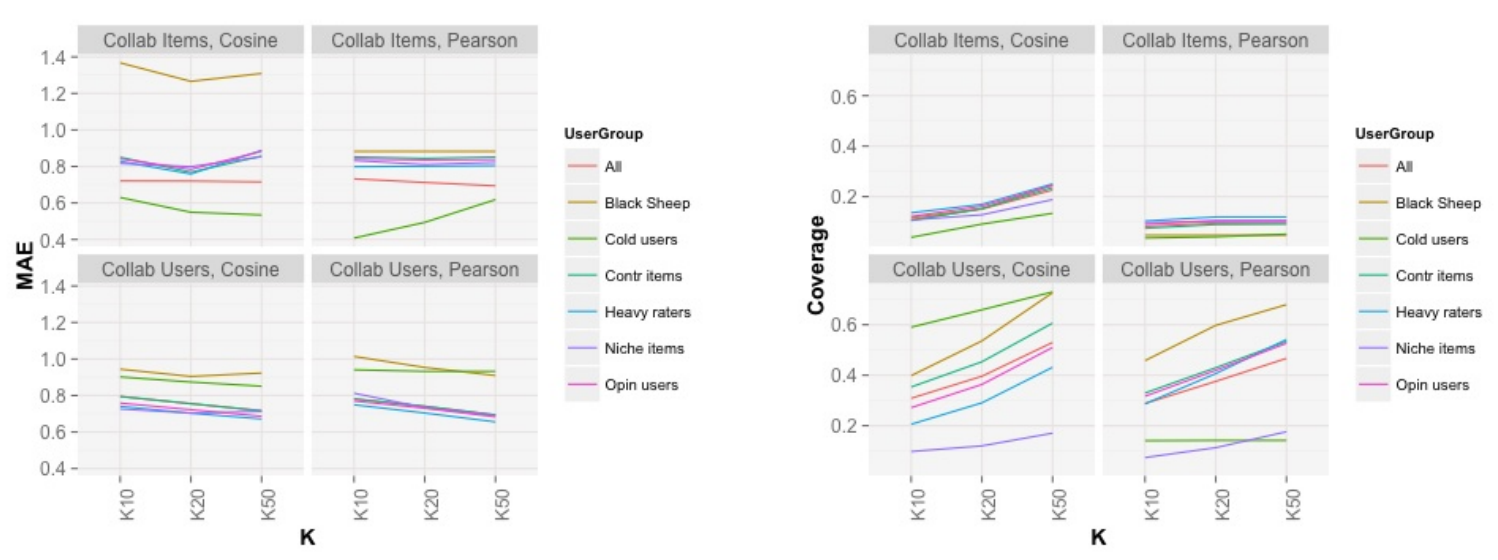

Figure 1: MAE and Coverage obtained with differents configurations of collaborative approach.

${ }^{2}$ http://www.trustlet.org/wiki/Epinions 
Now we analyse the results obtained with the approach based on trust. Figure 2 shows respectively the MAE and coverage obtained with the new proposal for different horizons. These figures show that a higher horizon value does not guarantee better results in MAE terms (in fact, the better MAE is obtained with $H=3$ ), but in coverage. Moreover, a higher horizon value penalizes the time to results as it implies much higher execution time.
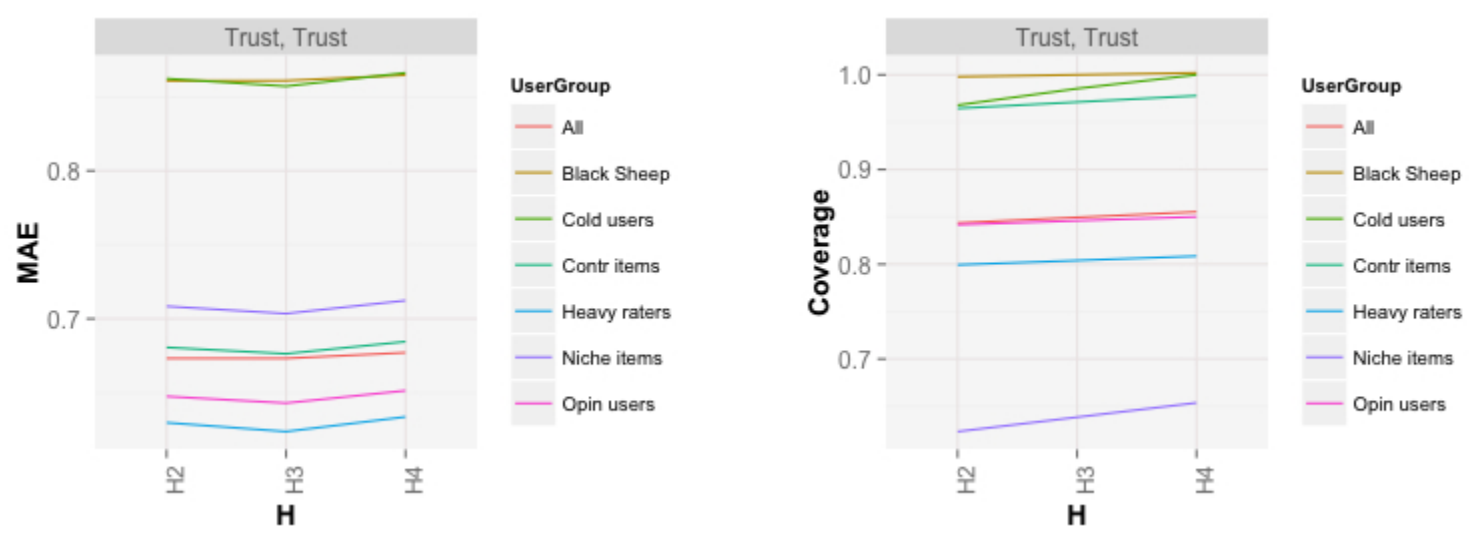

Figure 2: MAE and Coverage obtained with our suggested approach for differents horizons.

Figure 3 show the results of the comparison. We can see that the better MAE is obtained with item-based collaborative implementation for cold users, but only for very specific situations. However, in general terms, we see that the new proposal based on trust clearly outperforms the other approaches; specifically, we have achieved an improvement of $2.71 \%$. But, the best results of our proposal manifest in terms of coverage because it outperforms the other methods.
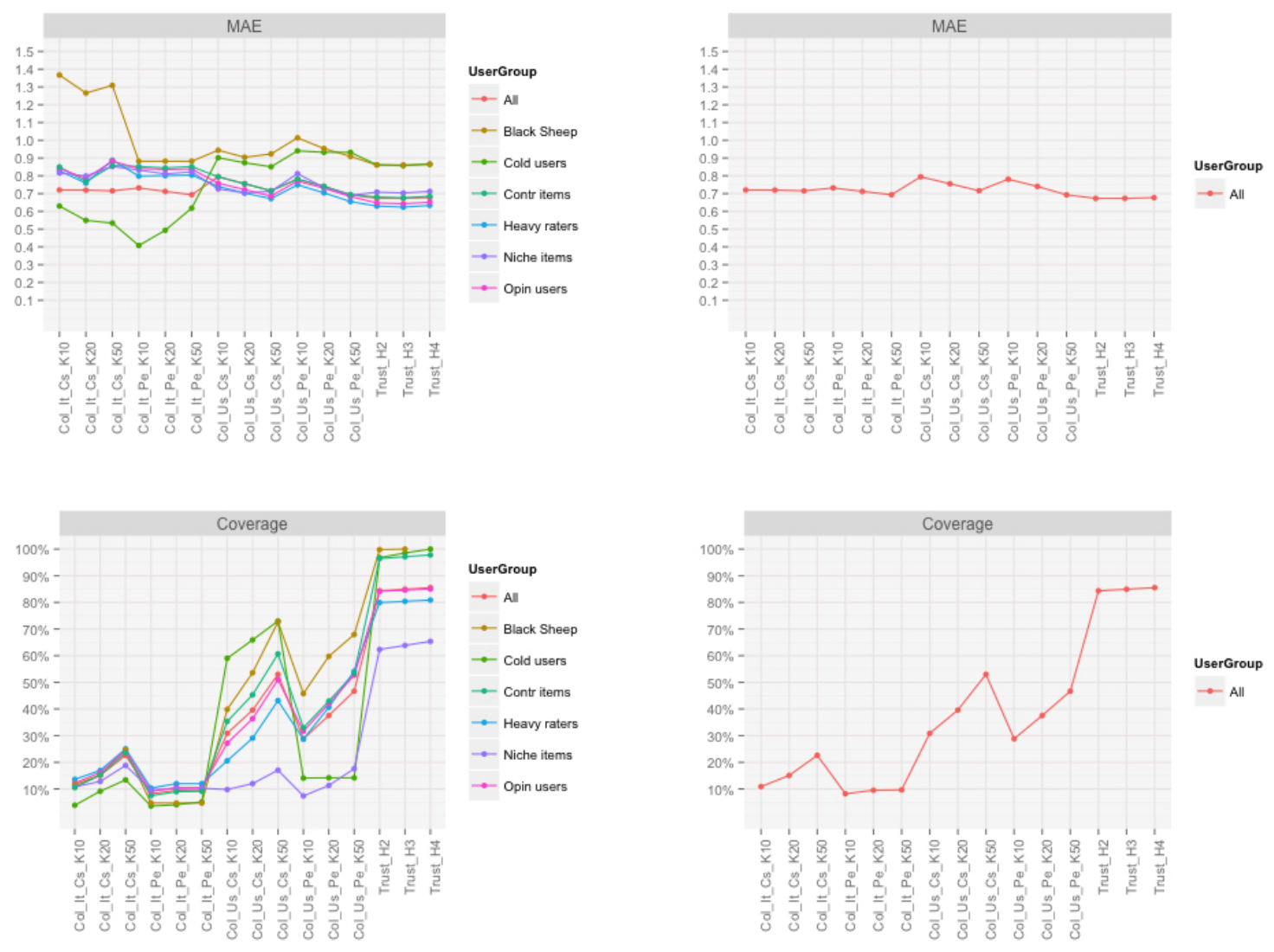

Figure 3: Comparison of MAE and coverage between new and the previous schemes. 


\section{Concluding remarks}

In this paper, we present a trust based fuzzy linguistic recommender system, to provide personalized activities to students in order to reinforce their individualized education. The main idea consists of not taking into account students with similar requirements or rating history, but rather trustworthy students. To achieve this, we have proposed a method to estimate the trust score between a pair of students. This system is applied in a real environment, providing personalized activities to students in the subjects Oral surgery I and II and Implantology II of Dentistry degree in the University of Granada (Spain). The main benefits of this system are the increase of the personalize degree of the education received by the students that also have the possibility of following the activities anywhere and anytime. We have evaluated the proposal, and the experimental results demonstrate the good results of the usage of the system as well as the perception by the students by enhancing the effectiveness of professors dealing with large group of students.

As future work, we consider to study the possibility of automatize the creation of activities by the system, based on individual feedback provided by the student of each component of the activities, as well as let the students create their own activities. Other proposal might be to focus on applying specific measures of the social networks analysis, exploiting the information represented in the trust network.

\section{Funding}

This paper has been developed with the FEDER financing under Project TIN2016-75850-R.

\section{Author contributions}

The authors contributed equally to this work.

\section{Conflict of interest}

The authors declare no conflict of interest.

\section{References}

[1] Burke, R. (2007). Hybrid Web Recommender Systems, P. Brusilovsky, A. Kobsa, and W. Nejdl (Eds.): The Adaptive Web, LNCS, 4321, 377-408, 2007.

[2] Burke, R.; Felfernig, A.; Göker, M.H. (2011). Recommender systems: An overview, Artificial Intelligence Magazine, 32(3), 13-18, 2011.

[3] Dascalu, M.I.; Bodea, C.N.; Moldoveanu, A.; Mohora, A.; Lytras, M.; Ordoñez de Pablos, P. (2015). A recommender agent based on learning styles for better virtual collaborative learning experiences, Computers in Human Behavior, 45, 243-253, 2015.

[4] Goga, M.; Kuyoro, S.; Goga, N. (2015). A recommender for improving the student academic performance, Procedia - Social and Behavioral Sciences, 180, 1481-1488, 2015.

[5] Golbeck, J.A. (2005). Computing and applying trust in web-based social networks, University of Maryland at College Park, 2005.

[6] Herrera, F.; Martínez, L. (2000). A 2-tuple fuzzy linguistic representation model for computing with words, IEEE Transactions on Fuzzy Systems, 8(6), 746-752, 2000.

[7] Herrera, F.; Martínez, L. (2001). A model based on linguistic 2-tuples for dealing with multigranularity hierarchical linguistic contexts in multiexpert decision- making, IEEE Transactions on Systems, Man and Cybernetics. Part B: Cybernetics, 31(2), 227-234, 2001. 
[8] Herrera-Viedma, E.; Pasi, G.; López-Herrera, A.G.; Porcel, C. (2006). Evaluating the Information Quality of Web Sites: A Qualitative Methodology Based on Fuzzy Computing With Words, Journal of the American Society for Information Science and Technology, 57(4), 538-549, 2006.

[9] Mata, F.; Martínez, L.; Herrera-Viedma, E. (2009). An Adaptive Consensus Support Model for Group Decision Making Problems in a Multi-Granular Fuzzy Linguistic Context, IEEE Transactions on Fuzzy Systems, 17(2), 279-290, 2009.

[10] Massa, P.; Avesani, P. (2009) Trust metrics in recommender systems, Computing with Social Trust, Springer, 259-285, 2009.

[11] Money, W.H.; Dean, B.P. (2019). Incorporating student population differences for effective online education: A content-based review and integrative model, Computers \& Education, 138, 57-82, 2019 .

[12] Morente-Molinera, J.A.; Pérez, I.J.; Ureña, R.; Herrera-Viedma, E. (2015). On multi-granular fuzzy linguistic modelling in group decision making problems: a systematic review and future trends, Knowledge Based Systems, 74, 49-60, 2015.

[13] Page, L.; Brin, S.; Motwani, R.; Winograd, T. (1998). The pagerank citation ranking: Bringing order to the web, Technical report, Stanford, USA, 1998.

[14] Porcel, C.; Ching-López, A.; Lefranc, G.; Loia, V.; Herrera-Viedma, E. (2018). Sharing notes: An academic social network based on a personalized fuzzy linguistic recommender system, Engineering Applications of Artificial Intelligence, 75, 1-10, 2018.

[15] Refaeilzadeh, P.; Tang, L.; Liu, H. (2008). Cross-Validation, http://www.public.asu.edu/ ltang9/papers/ency-cross-validation.pdf, 2008.

[16] Segal, D.; Gal, K.; Shani, G.; Shapira, B. (2019). A difficulty ranking approach to personalization in E-learning, International Journal of Human-Computer Studies, 130, 261-272, 2019.

[17] Shani, G.; Gunawardana, A. (2011). Evaluating Recommendation Systems, In: Ricci, F.; Rokach, L.; Shapira, B.; Kantor, P.B. (Eds.), Recommender Systems Handbook, Springer, 257-298, 2011.

[18] Sinha, R.R.; Swearingen, K. (2001). Comparing Recommendations Made by Online Systems and Friends, DELOS Workshop: Personalisation and Recommender Systems in Digital Libraries, 2001.

[19] Tan, H.C. (2019). Using a structured collaborative learning approach in a case-based management accounting course, Journal of Accounting Education, 49, 100638, 2019.

[20] Tejeda-Lorente, A.; Porcel, C.; Peis, E.; Sanz, R.; Herrera-Viedma, E. (2014). A quality based recommender system to disseminate information in a University Digital Library, Information Science, 261, 52-69, 2014.

[21] Victor, P.; Cornelis, C.; DeCock, M.; Pinheiro da Silva, P. (2009). Gradual trust and distrust in recommender systems, Fuzzy Sets and Systems, 160(10), 1367-1382, 2009.

[22] Zadeh, L.A. (1975). The Concept of a Linguistic Variable and Its Applications to Approximate Reasoning. Information Sciences, Part I, Information Sciences, 8, 199-249, Part II, Information Sciences, 8, 301-357, Part III, Information Sciences, 9, 43-80, 1975. 


\section{(c) (i) (8)}

Copyright (C)2020 by the authors. Licensee Agora University, Oradea, Romania.

This is an open access article distributed under the terms and conditions of the Creative Commons Attribution-NonCommercial 4.0 International License.

Journal's webpage: http://univagora.ro/jour/index.php/ijccc/

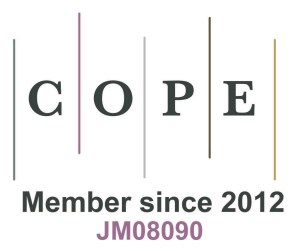

This journal is a member of, and subscribes to the principles of, the Committee on Publication Ethics (COPE).

https://publicationethics.org/members/international-journal-computers-communications-and-control

Cite this paper as:

Porcel, C.; Herce-Zelaya, J.; Bernabé-Moreno, J.; Tejeda-Lorente, A.; Herrera-Viedma, E. (2020). Trust Based Fuzzy Linguistic Recommender Systems as Reinforcement for Personalized Education in the Field of Oral Surgery and Implantology, International Journal of Computers Communications \& Control, 15(3), 3858, 2020.

https://doi.org/10.15837/ijccc.2020.3.3858 